\title{
ALTA OCORRÊNCIA DE GOTA CITOPLASMÁTICA DISTAL ALTERA PARÂMETROS RELACIONADOS AO MOVIMENTO ESPERMÁTICO DE REPRODUTORES SUÍNOS
}

\author{
(High occurrence of distal cytoplasmic droplet changes spermatic movement \\ parameters of boars)
}

Thais Schwarz Gaggini*, Helomar Barbosa, Marcelo Emílio Beletti, Fernanda Marcondes de Rezende, Robson Carlos Antunes.

\section{Universidade Federal de Uberlândia, Uberlândia, MG, Brasil}

\section{*Autor para correspondência: tsgaggini@yahoo.com.br}

RESUMO - O objetivo da pesquisa foi comparar parâmetros relacionados ao movimento espermático de ejaculados aprovados e reprovados exclusivamente devido à alta ocorrência de gota citoplasmática distal. Foram selecionadas 169 amostras de ejaculados para serem utilizadas no estudo. Destas, 25 foram reprovadas devido à presença de mais de $10 \%$ de gota distal e 144 foram aprovadas em todos os parâmetros avaliados. A avaliação da morfologia espermática foi realizada, contabilizando 200 células por amostra, utilizando microscópio com aumento de 100x. Foram considerados aprovados os ejaculados que apresentaram, de forma cumulativa, menos de $20 \%$ de células anormais, menos de $5 \%$ de defeitos de cabeça, acrossoma, colo e peça intermediária e menos de $10 \%$ de alterações de cauda e presença de gota proximal e distal. Características da motilidade espermática, avaliadas pelo sistema computadorizado, foram obtidas de todos os ejaculados avaliados no estudo. Ejaculados reprovados devido à alta ocorrência de gota distal apresentaram menor velocidade em linha reta $(p=0,04)$, menor motilidade total $(p=0,035)$ e progressiva $(p=0,004)$ quando comparados a ejaculados aprovados no exame morfológico. Os resultados indicam que a alta ocorrência de gota citoplasmática distal influencia nos parâmetros de movimento espermático de reprodutores suínos.

Palavras-chave: Duroc; ejaculado; morfologia espermática; motilidade espermática.

ABSTRACT - The aim of this study was to compare spermatic movement parameters of ejaculates approved and rejected exclusively due to high occurrence of distal cytoplasmic droplet. One-hundred-sixty-nine semen samples were selected to be used in the study. Of these, 25 were rejected due to the presence of more than $10 \%$ of distal droplets and 144 were approved in all evaluated parameters. Morphological examination was performed by counting 200 cells per sample using microscope at 100x magnification. Ejaculates that presented cumulatively less than $20 \%$ of abnormal cells, less than $5 \%$ of head defect, acrosome, neck and mid-piece defect and less than $10 \%$ of tail abnormality and occurrence of proximal and distal droplets were considered approved. Information of sperm motility characteristics evaluated by computer system was collected of all ejaculates evaluated by morphological test. Rejected ejaculates due high occurrence of distal cytoplasmic droplets presented lower velocity straight line $(p=0.04)$ and lower total $(p=0.035)$ and progressive $(p=0.004)$ motilities. These results indicate that high occurrence of distal droplets influence in spermatic movement parameters of boars.

Palavras-chave - Duroc; ejaculate; sperm morphology; sperm motility. 


\section{INTRODUÇÃO}

A presença de espermatozoides morfologicamente anormais no ejaculado é um indicativo de não conformidade no processo de formação e maturação do espermatozoide, ou ainda, no processamento do ejaculado, preparação e armazenamento de doses inseminantes (Althouse, 1998). As alterações dos espermatozoides observadas no exame de morfologia espermática podem ser denominadas como alterações primárias, secundárias ou terciárias. As primárias consistem em defeitos de cabeça, acrossoma, colo, peça intermediária e cauda. As secundárias são relacionadas às que ocorrem no epidídimo, sendo representadas principalmente pela presença de gota citoplasmática. As terciárias, que são as iatrogênicas, são causadas em casos de erro de preparação da amostra ou alterações nas condições ambientais, sendo representadas por alterações na cauda, cabeça normal desprendida e acrossoma destacado (Bonet et al., 2012).

As alterações primárias ocorrem em menor proporção quando comparadas às secundárias e terciárias (Waberski et al., 1994), sendo as gotas citoplasmáticas comumente observadas durante a avaliação morfológica (Briz e Fàbrega, 2013). Essas podem ser notadas em duas posições na peça intermediária e, dessa forma, são classificadas como gota proximal, quando próxima ao colo e cabeça, e gota distal, quando presente na porção terminal da peça intermediária. A gota citoplasmática distal é considerada menos importante do que a proximal (Althouse, 1998), em termos de comprometer a fertilidade in vivo e in vitro do reprodutor. Por este motivo, diversos manuais técnicos, livros e estudos consideram esta alteração sem significado patológico, não indicando a reprovação de reprodutores que apresentarem alta ocorrência desta no exame morfológico (Fonseca et al,. 1992; Bortolozzo et al., 2005; GonzálezUrdiáles et al. 2006; Bonet et al., 2013; CBRA, 2013).

Estudos indicam que a presença da gota citoplasmática na peça intermediária pode causar aumento de tamanho e flexão da cauda (Fentic et al., 2006; Cooper, 2011) e, consequentemente, prejudicar o movimento da célula espermática (Regan et al., 2012), reduzindo a sua capacidade fecundante. As pesquisas conduzidas referentes à importância das gotas citoplasmáticas abrangem os dois tipos de gotas de forma conjunta (Regan et al., 2012), não identificando o efeito isolado da gota citoplasmática distal nos parâmetros relacionados ao ejaculado e à fertilidade do reprodutor. A fim de verificar se a reprovação do ejaculado devido à alta porcentagem de gota citoplasmática distal deve ser considerada em centrais de inseminação artificial, o presente estudo comparou os parâmetros relacionados ao movimento espermático de ejaculados considerados aprovados e reprovados devido à alta ocorrência de gota citoplasmática distal no exame morfológico.

\section{MATERIAL E MÉTODOS}

Foram utilizados ejaculados de 132 reprodutores suínos de mesma linhagem genética (Duroc), sexualmente maduros, com idade média de 15,93 \pm 6,49 meses, intervalo de coleta médio de 5,77 $\pm 3,55$ dias, alojados na mesma central de inseminação artificial (Greenhill Al Stud, Saskatchewan Canadá) e submetidos ao mesmo manejo alimentar e de coleta de sêmen (método automatizado, Collectis ${ }^{\circledR}, \mathrm{IMV}$ ). O manejo dos animais na central de inseminação artificial foi realizado de acordo com o Código de Bem-Estar Animal Canadense (NFACC, 2014). 
Cento e sessenta e nove ejaculados foram avaliados quanto à morfologia espermática, sendo que destes, 144 foram aprovados em todos os parâmetros avaliados e 25 foram reprovados exclusivamente devido à presença de mais de $10 \%$ de gota citoplasmática distal. As análises foram realizadas por um técnico de laboratório previamente treinado e adaptado à rotina de avaliações da central de inseminação artificial, no período de outubro de 2015 e março de 2016.

Para a preparação das amostras para avaliação da morfologia espermática, $20 \mu \mathrm{L}$ de sêmen foram adicionados a $1 \mathrm{~mL}$ de formol citrato pré-aquecido à $36^{\circ} \mathrm{C}$, no momento do processamento do ejaculado. Uma gota da amostra $(20 \mu \mathrm{L})$, à temperatura ambiente, foi depositada sobre lâmina e lamínula e sua avaliação foi realizada utilizando microscópio de contraste de fase, com aumento de 100x e óleo de imersão. Duzentas células foram contadas e classificadas de acordo com a morfologia espermática: normal, gota citoplasmática proximal, gota citoplasmática distal, cauda enrolada, cauda dobrada, cabeça anormal, defeito de acrossoma, defeito de peça intermediária e de colo. Foram considerados aprovados os ejaculados que apresentaram, de forma cumulativa, menos de $20 \%$ de espermatozoides anormais, menos de $5 \%$ de defeitos de cabeça, acrossoma, colo e peça intermediária e menos de $10 \%$ de alterações de cauda e presença de gota citoplasmática proximal e distal.

As análises da cinética espermática foram realizadas por meio de sistema computadorizado de análise seminal (CASA; AndroVision ${ }^{\circledR}$, Minitüb $\mathrm{GmbH}$ ). Para a avaliação, uma alíquota do ejaculado in natura, previamente homogeneizada, foi diluída em Beltisville Thawing Solution (BTS), na proporção de 1:9 (90 $\mu \mathrm{L}$ do ejaculado e $810 \mu \mathrm{L}$ de
BTS pré-aquecido a $35^{\circ} \mathrm{C}$ ). A diluição foi realizada em microtubos com $2 \mathrm{~mL}$ de capacidade, utilizando pipeta eletrônica. Estes foram homogeneizados cinco vezes antes da avaliação e $3 \mu \mathrm{L}$ da solução foram introduzidos por capilaridade em câmara de contagem (20 $\mu \mathrm{m}$ profundidade, 4 câmaras de contagem) pré-aquecida a $36^{\circ} \mathrm{C}$. As amostras foram analisadas individualmente, em microscópio com 20x de aumento. Foram avaliados oito campos por amostra, seguindo direção retilínea de borda a borda da câmara Leja $^{\circledR}$, de forma que não houvesse repetição dos campos avaliados.

Os parâmetros obtidos a partir de cada ejaculado foram: motilidade espermática total, motilidade espermática progressiva (deslocamento maior do que $4,5 \mu \mathrm{m}$ percorridos em linha reta), amplitude do deslocamento lateral da cabeça (ALH), amplitude do deslocamento lateral da cabeça em relação à trajetória média (BCF número de vezes que a cabeça cruza a direção do movimento), distância percorrida em linha reta (DSL), distância média percorrida (DAP), distância percorrida na trajetória real do espermatozoide (DCL), velocidade média percorrida (VAP), velocidade em linha reta (VSL) e velocidade curvilínea (VCL - velocidade da trajetória real do espermatozoide).

Todos os parâmetros foram analisados quanto à normalidade pelo teste Shapiro-Wilk. As variáveis porcentagem de gota distal, motilidade total, motilidade progressiva, DSL, VAP, VSL e VCL foram analisadas por meio de análise não paramétrica, utilizando o Teste de Wilcoxon. As variáveis ALH, BCF, DAP e DCL foram analisadas utilizando o Teste T. As análises foram realizadas no programa estatístico Action, versão 2.8 (Estatcamp, 2014). A significância estatística foi considerada quando $p<0,05$. 


\section{RESULTADOS E DISCUSSÃO}

A estatística descritiva das alterações morfológicas observadas nas amostras de sêmen dos grupos considerados aprovado e reprovado encontra-se na Tabela 1. As variáveis porcentagem de gota distal, motilidade total, motilidade progressiva, DSL, VAP, VSL e VCL não apresentaram distribuição normal $(p<0,05)$ e, por este motivo foram analisadas por meio de análise não paramétrica. As variáveis ALH, BCF, DAP e DCL apresentaram distribuição normal $(p>0,05)$ e foram analisadas utilizando 0 Teste $T$. Ejaculados reprovados no exame morfológico devido à presença de mais de $10 \%$ de gota distal apresentaram menor motilidade total $(p=0,035)$, motilidade progressiva $(p=0,004)$ e velocidade em linha reta (VSL; $p=0,04$ ) do que ejaculados aprovados no exame morfológico (Tabela 2).

Tabela 1 - Anormalidades espermáticas observadas no grupo de reprodutores suínos aprovados e reprovados exclusivamente por presença de gota citoplasmática distal no exame morfológico.

\begin{tabular}{lll}
\hline Parâmetro & Ejaculados aprovados (\%) & Ejaculados reprovados (\%) \\
& $N=144$ & $N=25$
\end{tabular}

${ }^{5} \mathrm{PI}=$ peça intermediária

O efeito da motilidade total vem sendo estudado mesmo antes da utilização do CASA (Flowers, 1997) e a importância deste parâmetro na fertilidade é bastante conhecido, sendo correlacionados positivamente (Vyt et al., 2008; Kummer et al., 2013; Broekhuijse et al., 2012; Lima et al., 2015; Flowers et al., 2016).
Tabela 2 - Parâmetros espermáticos de ejaculados de reprodutores suínos considerados aprovados e reprovados exclusivamente por mais de $10 \%$ de gota distal no exame morfológico.

\begin{tabular}{|c|c|c|c|c|c|c|c|c|c|}
\hline & \multicolumn{4}{|c|}{$\begin{array}{l}\text { Ejaculados aprovados } \\
\left(\leq 10 \% \mathrm{GD}^{1}\right)\end{array}$} & \multicolumn{4}{|c|}{$\begin{array}{l}\text { Ejaculados reprovados } \\
\left(>10 \% \mathrm{GD}^{1}\right)\end{array}$} & \multirow{2}{*}{$p$} \\
\hline & $\begin{array}{l}\mathrm{Mi} \\
\mathrm{n}^{12}\end{array}$ & $\underset{13}{\operatorname{Md}}$ & $\operatorname{Max}_{14}$ & $\begin{array}{l}\mathrm{Me} \\
\mathrm{d}^{15}\end{array}$ & $\begin{array}{l}\mathrm{Mi} \\
\mathrm{n}\end{array}$ & Md & Max & Med & \\
\hline $\mathrm{GD}^{1}$ & $\begin{array}{l}0,0 \\
0\end{array}$ & $\begin{array}{l}3,2 \\
5\end{array}$ & $\begin{array}{l}10 \\
00\end{array}$ & $\begin{array}{l}4,0 \\
0\end{array}$ & $\begin{array}{l}11 \\
00\end{array}$ & $\begin{array}{l}15 \\
00\end{array}$ & $\begin{array}{l}53 \\
00\end{array}$ & $\begin{array}{l}20,3 \\
4\end{array}$ & $\begin{array}{l}<0,00 \\
01\end{array}$ \\
\hline $\begin{array}{l}\text { Mot } \\
\mathrm{T}^{2^{*}}\end{array}$ & $\begin{array}{l}73 \\
10\end{array}$ & $\begin{array}{l}88 \\
89\end{array}$ & $\begin{array}{l}97 \\
46\end{array}$ & $\begin{array}{l}88 \\
18\end{array}$ & $\begin{array}{l}72 \\
16\end{array}$ & $\begin{array}{l}87 \\
65\end{array}$ & $\begin{array}{l}94 \\
01\end{array}$ & $\begin{array}{l}85,8 \\
7\end{array}$ & 0,035 \\
\hline $\begin{array}{l}\text { Mot } \\
\mathrm{P}^{3^{*}}\end{array}$ & $\begin{array}{l}48 \\
56\end{array}$ & $\begin{array}{l}78 \\
55\end{array}$ & $\begin{array}{l}91 \\
40\end{array}$ & $\begin{array}{l}76 \\
70\end{array}$ & $\begin{array}{l}45 \\
72\end{array}$ & $\begin{array}{l}70 \\
24\end{array}$ & $\begin{array}{l}86 \\
88\end{array}$ & $\begin{array}{l}70,7 \\
1\end{array}$ & 0,004 \\
\hline$\underset{4^{*}}{\mathrm{DSL}}$ & $\begin{array}{l}13 \\
65\end{array}$ & $\begin{array}{l}18, \\
32\end{array}$ & $\begin{array}{l}29 \\
37\end{array}$ & $\begin{array}{l}19 \\
06\end{array}$ & $\begin{array}{l}13 \\
49\end{array}$ & $\begin{array}{l}16 \\
33\end{array}$ & $\begin{array}{l}21 \\
83\end{array}$ & $\begin{array}{l}17,6 \\
7\end{array}$ & 0,13 \\
\hline $\begin{array}{l}\text { VA } \\
P^{5^{*}}\end{array}$ & $\begin{array}{l}31 \\
00\end{array}$ & $\begin{array}{l}47 \\
00\end{array}$ & $\begin{array}{l}76 \\
02\end{array}$ & $\begin{array}{l}47 \\
72\end{array}$ & $\begin{array}{l}34 \\
00\end{array}$ & $\begin{array}{l}45 \\
50\end{array}$ & $\begin{array}{l}71 \\
24\end{array}$ & $\begin{array}{l}48,2 \\
7\end{array}$ & 0,60 \\
\hline$\underset{6^{*}}{\mathrm{VCL}}$ & $\begin{array}{l}40 \\
57\end{array}$ & $\begin{array}{l}62, \\
70\end{array}$ & $\begin{array}{l}126 \\
, 74\end{array}$ & $\begin{array}{l}67 \\
12\end{array}$ & $\begin{array}{l}45 \\
29\end{array}$ & $\begin{array}{l}65 \\
87\end{array}$ & $\begin{array}{l}123 \\
, 18\end{array}$ & $\begin{array}{l}74,9 \\
4\end{array}$ & 0,42 \\
\hline \multirow[t]{2}{*}{$\underset{7^{\star}}{\operatorname{VSL}}$} & $\begin{array}{l}29 \\
02\end{array}$ & $\begin{array}{l}71, \\
00 \\
\end{array}$ & $\begin{array}{l}83, \\
00 \\
\end{array}$ & $\begin{array}{l}62, \\
42 \\
\end{array}$ & $\begin{array}{l}29 \\
27\end{array}$ & $\begin{array}{l}53, \\
84\end{array}$ & $\begin{array}{l}74, \\
00\end{array}$ & $\begin{array}{l}53,2 \\
9 \\
\end{array}$ & 0,04 \\
\hline & Med & & $\mathrm{DP}^{16}$ & & Med & & DP & & $\begin{array}{l}p- \\
\text { valor }\end{array}$ \\
\hline $\begin{array}{l}\text { ALH } \\
8 a\end{array}$ & 2,82 & & 0,51 & & 2,90 & & 0,64 & & 0,63 \\
\hline $\begin{array}{l}B C \\
F^{9 a}\end{array}$ & 26,18 & & 4,55 & & 25,73 & & 5,05 & & 0,15 \\
\hline $\begin{array}{l}\text { DA } \\
\mathrm{P}^{10 \alpha}\end{array}$ & 26,17 & & 4,55 & & 25,73 & & 5,04 & & 0,75 \\
\hline $\begin{array}{l}D C \\
L^{11 \alpha}\end{array}$ & 42,65 & & 8,27 & & 43,64 & & 9,65 & & 0,70 \\
\hline
\end{tabular}

a Comparação utilizando o Teste $T(p<0,05)$

${ }^{1} \mathrm{GD}$ - gota distal (\%); ${ }^{2}$ MotT - motilidade espermática total (\%); ${ }^{3}$ MotP - motilidade espermática progressiva (\%); ${ }^{4} \mathrm{ALH}$ - amplitude do deslocamento lateral da cabeça $(\mu \mathrm{m}) ;{ }^{5} \mathrm{BCF}$ - amplitude do deslocamento lateral da cabeça em relação à trajetória média $(\mathrm{Hz})$; ${ }^{6} \mathrm{DAP}$ - distância média percorrida $(\mu \mathrm{m}) ;{ }^{7} \mathrm{DCL}$ - distância percorrida na trajetória real do espermatozoide $(\mu \mathrm{m}) ;{ }^{8} \mathrm{DSL}$ - distância percorrida em linha reta $(\mu \mathrm{m}) ;{ }^{9} \mathrm{VAP}$ - velocidade média percorrida $(\mu \mathrm{m} / \mathrm{seg}) ;{ }^{10} \mathrm{VCL}$ velocidade curvilínea $(\mu \mathrm{m} / \mathrm{seg}) ;{ }^{11} \mathrm{VSL}$ - velocidade em linha reta $(\mu \mathrm{m} / \mathrm{seg}) ;{ }^{12} \mathrm{Min}$ - valor mínimo; ${ }^{13} \mathrm{Md}$ - mediana; ${ }^{14} \mathrm{Max}$ - valor máximo ${ }^{15} \mathrm{Med}$ - média; ${ }^{16} \mathrm{DP}$ - desvio padrão.

As pesquisas avaliando a motilidade progressiva são mais recentes, porém, demonstram uma correlação positiva desta variável com a taxa de parto (Broekhuijse et al,. 2012), sendo um indicador importante de fertilidade de reprodutores (Kummer et al., 2013). Dessa forma, a influência negativa da gota distal na motilidade espermática total e progressiva, observada no presente estudo, pode atuar como um fator limitante sobre a fertilidade do reprodutor.

As variáveis relacionadas à velocidade e ao deslocamento das células espermáticas vêm sendo estudadas, mas não se sabe ao certo se todos os parâmetros obtidos pelo CASA estão envolvidos no processo de fecundação (Didion, 2008). As pesquisas envolvendo esses 
parâmetros apresentam resultados variáveis, porém, diversos estudos relatam correlação positiva de VAP, VSL (Flowers et al., 2016), ALH, BCF e VCL (Kummer et al. 2013) com fertilidade e, de ALH, BCF, VCL, VAP e VSL, com tamanho de leitegada (Holt et al.1997). Broekhuijse et al. (2012), que fizeram análises diferenciadas dos parâmetros relacionados ao movimento espermático, observaram que VCL e BCF juntos explicaram $9 \%$ da variação na taxa de parto. Estes indicadores apresentaram correlação negativa ( $r=-$ 0,37 e $r=-0,72$, respectivamente), o que indica que quanto maior esses parâmetros, menor a taxa de parto do plantel. VAP e VSL, que foram correlacionadas positivamente com 0 número de nascidos totais, e $\mathrm{ALH}$, que foi correlacionado negativamente, explicaram juntos $10 \%$ da variação desta variável.

No presente estudo, a maior ocorrência de gota distal não afetou os valores de VAP, VCL, ALH e BCF, mas diferiu entre os grupos testados, apresentando menor valor de VSL para amostras reprovadas. $O$ efeito negativo da alta ocorrência de gota citoplasmática distal no parâmetro VSL pode ser justificado pelo fato de que esta anormalidade morfológica acarreta alterações no movimento espermático (Regan et al., 2012).

\section{CONCLUSÃO}

A alta ocorrência de gota citoplasmática distal influencia negativamente na motilidade total, progressiva e velocidade em linha reta dos espermatozoides e, por este motivo, este parâmetro deve ser considerado como causa de reprovação na avaliação de ejaculados suínos.

\section{REFERÊNCIAS}

ALTHOUSE, G.C. Cytoplasmic droplets on boar sperm cells. Swine Health and Production, v.6, n.3, p.128, 1998.

BONET, S.; BRIZ, M.D.; YESTE, M. A Proper assessment of boar sperm function may not only require conventional analyses but also others focused on molecular markers of epididymal maturation. Reproduction in Domestic Animals, v.47, p.52-64, 2012.

BONET, S.; GARCIA, E. SEPÚLVEDA, $\mathrm{L}$. The boar reproductive system. In: BONET, S.; CASAS, I.; HOLT, W.V. et al. Boar reproduction: fundamentals and new biotechnological trends. Berlin: Springer-Verlag, 2013, p.65-107. BORTOLOZZO, F.P.; WENTZ, I.; FERREIRA, F.M. et al. Exame do ejaculado. In: BORTOLOZZO, F.P.; WENTZ, I.; BENNEMANN, P.E. et al. Inseminação artificial na suinocultura tecnificada. Porto Alegre: Pallotti, 2005, cap.7, p.69-89.

BRIZ, M.D.; FÁBREGA, A. Boar spermatozoa within the male genital tract, the boar spermatozoon. In: BONET, S.; CASAS, I.; HOLT, W.V. et al. Boar reproduction: fundamentals and new biotechnological trends. Berlin: Springer-Verlag, 2013, part I, p. 3-47.

BROEKHUIJSE, M.L.W.J.; SOSTARIC, E.; FEITSMA, $H$. et al. Application of computer-assisted semen analysis to explain variations in pig fertility. Journal of Animal Science, v.90, p.779-789, 2012.

COLÉGIO BRASILEIRO DE REPRODUÇÃO ANIMAL - CBRA. Manual para exame andrológico e 
avaliação de sêmen animal. $3^{\mathrm{a}} \mathrm{ed}$. Belo Horizonte: CBRA, 2013, 104p.

COOPER, T.G. The epididymis, cytoplasmic droplets and male fertility. Asian Journal of Andrology, v.13, p.130-138, 2011.

DIDION, B.A. Computer-assisted semen analysis and its utility for profiling boar semen samples. Theriogenology, v.70, p.1375-1376, 2008.

ESTIENNE, M.J.; HARPER, A.F.; DAY, J.L. Characteristics of sperm motility in boar semen diluted in different extenders and stored for seven days at $18^{\circ} \mathrm{C}$. Reproductive Biology, v.7, n.3, 221-231, 2007.

FENTIC, S.; YEUNG, C.; SONNTAG, B. et al. Relationship of cytoplasmic droplets to motility, migration in mucus, and volume regulation of human spermatozoa. Journal of Andrology, v.27, p.294-301, 2006.

FLOWERS, W.L.; DELLER, F.; STEWART, K.R. Use of heterospermic inseminations and paternity testing to evaluate the relative contributions of common sperm traits and seminal plasma proteins in boar fertility. Animal Reproduction Science, v.174, p.123131, 2016.

FLOWERS, W.L. Management of boars for efficient semen production. Journal of Reproduction and Fertility, v.52, p.67-78, 1997.

FONSECA, V.O.; VALE FILHO, V.R.; MIES FILHO, A. et al. Espermiograma. In: FONSECA, V.O.; VALE FILHO, V.R.; MIES FILHO, A. et al. Procedimento para exame andrológico e avaliação de sêmen animal. 1eㅡ. Belo Horizonte: CBRA, 1992. p.6-30.

GONZÁLEZ-URDIÁLEZ, R.; TEJERINA, F.; DOMÍNGUES, J.C. et al. Técnicas de análisis rutinario de la calidad espermática: motilidad, vitalidad, concentración, resistencia osmótica y morfología espermática. In: BONET, S.; MARTÍNEZ, R.; RODRÍGUEZ, J.E. et al. Manual de técnicas de reproducción asistida en porcino. Girona: Universitat de Girona and Red Temática Nacional de Reproducción Porcina, 2006. p.1938.

HOLT, C.; HOLT, W.V.; MOORE, H.D.M. et al. Objectively measured boar sperm motility parameters correlate with outcomes of on-farm insemination: results of two fertility trials. Journal of Andrology, v.12, p.312-323, 1997.

JOHNSON, L.A.; WEITZ, K.F.; FISER, $P$. et al. Storage of boar semen. Animal Reproduction Science, v.62, p.143172, 2000.

KUMMER, A.B.H.P.; GAGGINI, T.S.; BERNARDI, M.L. et al. Multivariate analyses for determining the association of field porcine fertility with sperm motion traits analysed by computer-assisted semen analysis and with sperm morphology, Reproduction in Domestic Animals, v.48, p.747-754, 2013.

LIMA, D.M.A.; PINHO, R.O.; SIQUEIRA, J.B. et al. Correlation of sperm parameters with fertility in two commercial pig lines. Acta Scientiae Veterinariae, v.43, p.1-6, 2015.

NATIONAL FARM ANIMAL CARE CONCIL (NFACC). Canada' Codes of Practice for the Care and Handling of Pigs, 2014. Disponível em: <http://www.nfacc.ca/codes-ofpractice/pigs >. Acesso em 12/03/2018.

REGAN, A.K.; AGARWAL, A.; VAN DER LINDE, M. et al. An investigation of excess residual cytoplasm in human spermatozoa and its distinction from the cytoplasmic droplet. Reproduction 
Biology and Endocrinology, v.10, p.18, 2012.

RIBEIRO, C.V.; MORONI, J.L.; MENEGAT, M.B. et al. The influence o fair contact on the quality of extended boar semen. Acta Scientiae Veterinariae, v.44, 1-7, 2016.

VYT, P. MAES, D.; QUINTEN, C.; RIJSSELAERE, T. et al. Detailed motility examination of porcine semen and its predictive value towards reproductive performance in sows. Flemic Veterinary, v.77, p.291-298, 2008.

WABERSKI, F.; MEDING, S.; DIRKSEN, G. et al. Fertility of long-termstored boar semen: influence of extender (Androhep and Kiev), storage time and plasma droplets in the semen. Animal Reproduction Science, v.36, p.141$151,1994$. 